\title{
Can Patient-Provider Interpersonal Interventions Achieve the Quadruple Aim of Healthcare? A Systematic Review
}

\author{
Marie C. Haverfield, $P h D^{1,2}$ (D), Aaron Tierney, $B A^{3}$, Rachel Schwartz, $P h D^{1,2}$, \\ Michelle B. Bass, PhD, MSI, AHIP4, Cati Brown-Johnson, PhD ${ }^{5}$, Dani L. Zionts, MSCPH ${ }^{5}$, \\ Nadia Safaeinili, $M P H^{5}$, Meredith Fischer, $M A^{5}$, Jonathan G. Shaw, MD, $M S^{5}$, \\ Sonoo Thadaney, MBA ${ }^{3}$, Gabriella Piccininni, BS ${ }^{3}$, Karl A. Lorenz, MD, MS ${ }^{1,5}$, \\ Steven M. Asch, MD, MPH ${ }^{1,5}$, Abraham Verghese, $\mathrm{MD}^{3}$, and Donna M. Zulman, \\ $M D, M S^{1,5}$
}

\begin{abstract}
${ }^{1}$ Center for Innovation to Implementation (Ci2i), VA Palo Alto Health Care System (152-MPD), Menlo Park, CA, USA; ${ }^{2}$ Stanford University Center for Primary Care and Outcomes Research (PCOR) and Center for Health Research and Policy (CHRP), Stanford, CA, USA; ${ }^{3}$ School of Medicine, Stanford University, Stanford, CA, USA; ${ }^{4}$ Countway Library of Medicine, Harvard, Boston, CA, USA; ${ }^{5}$ Division of Primary Care and Population Health, Stanford University School of Medicine, Stanford, CA, USA.
\end{abstract}

BACKGROUND: Human connection is at the heart of medical care, but questions remain as to the effectiveness of interpersonal interventions. The purpose of this review was to characterize the associations between patientprovider interpersonal interventions and the quadruple aim outcomes (population health, patient experience, cost, and provider experience).

METHODS: We sourced data from PubMed, EMBASE, and PsycInfo (January 1997-August 2017). Selected studies included randomized controlled trials and controlled observational studies that examined the association between patient-provider interpersonal interventions and at least one outcome measure of the quadruple aim. Two abstractors independently extracted information about study design, methods, and quality. We characterized evidence related to the objective of the intervention, type and duration of intervention training, target recipient (provider-only vs. provider-patient dyad), and quadruple aim outcomes.

RESULTS: Seventy-three out of 21,835 studies met the design and outcome inclusion criteria. The methodological quality of research was moderate to high for most included studies; $67 \%$ of interventions targeted the provider. Most studies measured impact on patient experience; improvements in experience (e.g., satisfaction, patient-centeredness, reduced unmet needs) often corresponded with a positive impact on other patient health outcomes (e.g., quality of life, depression, adherence). Enhanced interpersonal interactions improved provider well-being, burnout, stress, and confidence in communicating with difficult patients. Roughly a quarter of studies evaluated cost, but the majority reported no significant

Electronic supplementary material The online version of this article (https://doi.org/10.1007/s11606-019-05525-2) contains supplementary material, which is available to authorized users.

Prior presentations: Academy Health Annual Research Meeting, June 2019

Received October 16, 2019

Revised October 16, 2019

Accepted October 25, 2019

Published online January 9, 2020 differences between intervention and control groups. Among studies that measured time in the clinical encounter, intervention effects varied. Interventions with lower demands on provider time and effort were often as effective as those with higher demands.

DISCUSSION: Simple, low-demand patient-provider interpersonal interventions may have the potential to improve patient health and patient and provider experience, but there is limited evidence that these interventions influence cost-related outcomes.

KEY WORDS: interpersonal interventions; quadruple aim; systematic review; patient-provider communication.

$\mathrm{J}$ Gen Intern Med 35(7):2107-17

DOI: $10.1007 / \mathrm{s} 11606-019-05525-2$

(C) Society of General Internal Medicine 2020

66 $\mathrm{Y}$ ou treat a disease, you win, you lose. You treat a person, I guarantee you, you'll win, no matter what the outcome." — Patch Adams.

\section{INTRODUCTION}

Humanism is central to clinical care, yet the demands of modern medicine and technology can interfere with meaningful interpersonal interactions between patients and providers. ${ }^{1,2}$ Beyond their inherent value, better connections between patients and providers can improve symptoms, increase adherence to medical recommendations, and reduce medical errors. ${ }^{3-5}$ Moreover, routinely failing to connect contributes to physician burnout, which may initiate a downward spiral in patient-provider relationships. ${ }^{6}$

Efforts to improve interpersonal patient-provider interactions have often used patient-centered approaches (e.g., shared decision-making) to enhance communication. ${ }^{7-10}$ Previous reviews have explored the effectiveness of 
resulting interventions, ${ }^{11,12}$ mostly focusing on how they might improve patient morbidity and satisfaction with care. ${ }^{13,14}$ Fewer studies have looked at the larger range of health-related outcomes including cost and the provider experience, or at which attributes effective interventions might have in common.

To fill this gap, we designed a systematic review to examine the association between patient-provider interpersonal interventions and the quadruple aim of improving population health, patient experience, cost of care, and provider experience. ${ }^{15}$ The first three outcome domains were originally proposed by Berwick and the Institute for Healthcare Improvement as the triple aim (improving the care of individuals, improving health of populations, and reducing per capita costs) and have become widely accepted as the pillars of optimizing healthcare performance. ${ }^{16}$ The addition of the fourth aim - provider experience - by Bodenheimer and Sinsky in 2014 recognizes that "the care of the patient requires care of the provider." 15 We sought to identify promising interventions - and characteristics across interventions - that have the greatest potential to enhance interpersonal interactions and meaningful outcomes.

\section{METHODS}

\section{Data Sources and Searches}

To ensure comprehensiveness, we searched PubMed, EMBASE, and PsycInfo in August 2017, published between January 1, 1997, and August 31, 2017.

\section{Study Selection}

We searched for studies of interpersonal interventions, defined as those that address the relationship between patients and their providers (e.g., doctor, nurse) and that incorporate or encourage "a selective, systemic process that allows people to reflect and build personal knowledge of one another and create shared meanings." ${ }^{" 17}$ To capture all such interventions, our search included a broad array of MeSH and keywords encompassing domains including trust, empathy, humanism, nonverbal communication, social skills, and interpersonal relations (see Table 4 in the Appendix for complete search strategies).

We defined the following a priori inclusion criteria: (1) the study was a randomized controlled trial (RCT), controlled observational study (i.e., an experimental study with a nonrandomized control group), or systematic review; (2) the study evaluated an interpersonal intervention (concept 1 search terms); and (3) the study included at least one quadruple aim outcome (i.e., an outcome pertaining to population health [operationalized as outcomes related to health or healthcare], patient experience, provider experience, or direct or indirect measures of cost) (concept 2 search terms, Table 4 in the
Appendix). ${ }^{15}$ We excluded non-English studies, animal research, and research with participants younger than 18 years of age.

To conduct the review, we utilized the online systematic review tool Covidence (Veritas Health Innovation Ltd., Melbourne, 2018). Throughout the review process, team members checked each article for eligibility based on study design (i.e., RCT, controlled observational study, systematic review), interpersonal intervention, and presence of at least one quadruple aim-related outcome reported (e.g., frequency of return visits, blood pressure, patient satisfaction, provider burnout). One reviewer (A.T.) examined all systematic reviews to capture additional eligible RCTs and controlled observational studies.

\section{Data Extraction and Quality Assessment}

Two or more authors independently reviewed each title/ abstract and the selected full-text articles (I.R., A.T., G.P., S.B., R.S.). Two reviewers independently abstracted (I.R., A.T., G.P., S.B.) and assessed the methodological quality of each study (A.T., S.B.) using the Cochrane criteria for grading randomized controlled trials and Effective Practice and Organisation of Care (EPOC) criteria for assessing risk of bias in observational studies. ${ }^{18}$ In addition, two reviewers independently graded the level of evidence for each study based on the Oxford Centre for Evidence-Based Medicine levels of evidence (A.T., M.H.). ${ }^{19}$ Disagreements were resolved through discussion and consensus with a third reviewer (M.H., R.S.).

\section{Data Synthesis and Analysis}

Given the heterogeneity of outcomes across studies, we were unable to conduct a meta-analysis and instead systematically organized the data in an effort to synthesize findings across studies. For each study, we characterized the intervention content focus, structure, demand on participants, and target (or recipient) of the intervention (i.e., provider, or provider and patient). For content focus, we categorized interventions into one of nine focus areas based on the concept most closely related to the intervention objective (e.g., motivational interviewing, communication skills, shared decision-making). For intervention structure, we determined whether the intervention involved any of four structures: (1) educational component (e.g., workshop or presentation), (2) practice (e.g., dyadic exercises such as role-playing and teach-back), (3) general instruction (i.e., recipient receives explicit instructions about the intervention with or without additional training), and/or (4) a tool (e.g., pocket guide, handout that encourages perspectivetaking). Because interventions often combined components, we developed nine mutually exclusive categories to encompass the observed combinations of these four structures. To assess the intervention demand on participants, we adapted the intensity rating of Rao et al. by examining the time spent in training based on actual training time and 
Table 1 Characteristics of Interventions Included in the Review

\begin{tabular}{lll}
\hline \hline & $\boldsymbol{n}$ & \% \\
\hline Content focus & & \\
Motivational interviewing & 6 & 8 \\
Health literacy & 3 & 4 \\
Patient-provider relationship & 3 & 4 \\
Patient-centered care & 14 & 19 \\
Communication skills & 21 & 29 \\
Shared decision-making & 5 & 7 \\
Specific communication technique & 16 & 22 \\
Psychological/therapeutic interview & 2 & 3 \\
Mindfulness & 3 & 4 \\
Structure* & 65 & 89 \\
Education & 60 & 82 \\
Practice & 6 & 8 \\
Instructions & 29 & 40 \\
Tool & & \\
Demand on provider time/effort & 24 & 33 \\
Low & 29 & 40 \\
Moderate & 12 & 16 \\
High & 8 & 11 \\
Not reported & & \\
Target recipient & 51 & 67 \\
Provider-only & 22 & 30 \\
Provider and patient &
\end{tabular}

*Interventions could include more than one structural component

duration of training period. ${ }^{20}$ We categorized each intervention as low, moderate, or high demand. The target (or recipient) of the intervention was categorized as provideronly or provider-patient dyad (Table 1).

We characterized study outcomes and mapped them to each of the quadruple aim domains (population health outcomes, patient experience, cost of care, and provider experience). Because RCTs rarely examine changes in population health, we examined health outcomes (e.g., physical health, mental health, treatment adherence, healthcare utilization, pain, and patient prescription rate) based on the assumption that changes in these outcomes have the potential to influence population health over time. Patient experience outcomes included satisfaction, provider communication quality, patient perception of partnership, extent of patient participatory decision-making, patient motivation to adhere, quality of health information provided, and perceived visit length. Cost outcomes included direct measures of cost and outcomes that could potentially generate savings (e.g., utilization efficiency, prescription rates). To examine reports of visit duration, we gathered all studies that account for visit length $(n=11)$ and abstracted information on the control/baseline visit length as well as identified reduction, extension, or no change in time. Provider experience outcomes included provider communication (perceived and observed), provider perception of relationship with patient, satisfaction, burnout/stress, and patient-centeredness (perceived and observed).

\section{Role of the Funding Source}

The funding sources had no role in the design and conduct of the study; collection, management, analysis, and interpretation of the data; preparation, review, or approval of the manuscript; and decision to submit the manuscript for publication. The review is registered at Prospero: CRD42019120160.

\section{RESULTS}

\section{Selected Articles and Study Characteristics}

Our initial search, title/abstract and full-text screening, and culling of studies from systematic reviews resulted in a total of 73 articles (see Table 2). ${ }^{21-93}$ Two articles reported data from the same trial, but the outcomes and timeframes were unique and are therefore referred to as separate studies. ${ }^{43,44}$ Sixty-seven studies were randomized controlled trials and six were controlled observational studies. Studies predominantly took place in the outpatient setting $(86 \%)$, with some occurring in the inpatient setting (8\%), or both (6\%). Most studies took place outside of the USA $(60 \%)$. Approximately two thirds of the studies (45/73) described a framework that guided the intervention. Study outcomes were assessed over a wide range of follow-up times (i.e., 2 days to 3.5 years, with an average of 258 days after the intervention). No studies reported outcomes beyond 3.5 years. Study characteristics are presented in Table 5 in the Appendix.

Quality Assessment and Levels of Evidence. The methodological quality of research was moderate to high for most studies. ${ }^{18}$ Most studies $(n=71 / 73)$ had a quality score above 2.0 , which indicates moderate to high quality, with an average score of $2.57(\mathrm{SD}=0.21)$. The primary reason for lower quality scores was incomplete outcome data. Consistent with the combined Cochrane and EPOC ratings, $92 \%$ of studies were level 1 according to the levels of evidence, reflecting the highest-quality research (Table 6 in the Appendix).

\section{Synthesis of Interpersonal Interventions}

Interventions most commonly focused on general communication skills (29\%; e.g., verbal and nonverbal skills), a specific communication technique $(22 \%$; e.g., instruction to ask a patient "Is there something else?" rather than "Is there anything else?" at the end of the visit), ${ }^{66}$ or a patient-centered care strategy (19\%; e.g., training based on a patient-centered care framework). Fewer interventions focused on motivational interviewing (8\%), shared decision-making $(7 \%)$, the patient-provider relationship (4\%), mindfulness (4\%), health literacy $(4 \%)$, or a psychological/therapeutic technique $(3 \%)$. In terms of demand on participants' time and effort, 24 (33\%) of the interventions were low demand, $29(40 \%)$ were moderate demand, and $12(16 \%)$ were high demand (Table 7 and 8 in the Appendix). Eight (11\%) studies did not report intervention time and effort demands. Most interventions incorporated more than one structural component, most commonly an educational activity (e.g., workshop) and a practice element (e.g., role-play) $(n=34,47 \%)$. Three interventions $(4 \%)$ were limited to a practice, three (4\%) were limited to instruction 
Table 2 Study and Intervention Characteristics

\begin{tabular}{|c|c|c|c|c|c|c|c|}
\hline$\overline{\text { Study }}$ & Study design* & $\begin{array}{l}\text { Quality } \\
\text { assessment }\end{array}$ & Content focus & $\begin{array}{l}\text { Intervention } \\
\text { structure }\end{array}$ & Demand & Recipient & $\begin{array}{l}\text { Framework } \\
\text { (yes/no) }\end{array}$ \\
\hline $\begin{array}{l}\text { Aboumatar } \\
2013^{21}\end{array}$ & $\mathrm{RCT}^{*}$ & 2.45 & Health literacy & $\begin{array}{l}\text { Education and } \\
\text { tool }\end{array}$ & Low & $\begin{array}{l}\text { Provider } \\
\text { and patient }\end{array}$ & No \\
\hline $\begin{array}{l}\text { Aiarzaguena } \\
2007^{22}\end{array}$ & $\mathrm{RCT}$ & 3.00 & $\begin{array}{l}\text { Specific } \\
\text { communication } \\
\text { technique }\end{array}$ & $\begin{array}{l}\text { Education, } \\
\text { practice, and tool }\end{array}$ & Moderate & Provider & Yes \\
\hline Ajam 2017(97) & $\mathrm{RCT}$ & 3.00 & $\begin{array}{l}\text { Communication } \\
\text { skills }\end{array}$ & $\begin{array}{l}\text { Education and } \\
\text { practice }\end{array}$ & High & Provider & Yes \\
\hline $\begin{array}{l}\text { Akturan } \\
2017(98)\end{array}$ & RCT & 2.82 & $\begin{array}{l}\text { Psychological/ } \\
\text { therapeutic interview }\end{array}$ & $\begin{array}{l}\text { Education and } \\
\text { tool }\end{array}$ & Low & Provider & Yes \\
\hline Alder $2007^{25}$ & $\mathrm{RCT}^{*}$ & 2.64 & $\begin{array}{l}\text { Communication } \\
\text { skills }\end{array}$ & $\begin{array}{l}\text { Education, } \\
\text { practice, and tool }\end{array}$ & Moderate & Provider & No \\
\hline Altiner 2007(99) & RCT & 2.67 & Health literacy & Practice & $\begin{array}{l}\text { Not } \\
\text { reported }\end{array}$ & $\begin{array}{l}\text { Provider } \\
\text { and patient }\end{array}$ & Yes \\
\hline Au $2012^{27}$ & $\mathrm{RCT}$ & 2.45 & $\begin{array}{l}\text { Specific } \\
\text { communication } \\
\text { technique }\end{array}$ & Tool & Low & $\begin{array}{l}\text { Provider } \\
\text { and patient }\end{array}$ & Yes \\
\hline $\begin{array}{l}\text { Aubin-Auger } \\
2016^{28}\end{array}$ & $\mathrm{RCT}^{*}$ & 2.36 & Patient-centered care & $\begin{array}{l}\text { Education and } \\
\text { practice }\end{array}$ & Low & Provider & Yes \\
\hline Bakker $2007^{29}$ & RCT & 2.64 & $\begin{array}{l}\text { Specific } \\
\text { communication } \\
\text { technique }\end{array}$ & Education & High & Provider & No \\
\hline Bashour $2013^{30}$ & $\mathrm{RCT}^{*}$ & 2.91 & $\begin{array}{l}\text { Communication } \\
\text { skills }\end{array}$ & $\begin{array}{l}\text { Education and } \\
\text { practice }\end{array}$ & Moderate & Provider & No \\
\hline Bellón $2008^{31}$ & $\mathrm{RCT}$ & 2.55 & $\begin{array}{l}\text { Specific } \\
\text { communication } \\
\text { technique }\end{array}$ & $\begin{array}{l}\text { Education, } \\
\text { practice, and tool }\end{array}$ & Moderate & Provider & Yes \\
\hline $\begin{array}{l}\text { Bernhard } \\
2012^{32}\end{array}$ & RCT* & 2.42 & $\begin{array}{l}\text { Specific } \\
\text { communication } \\
\text { technique }\end{array}$ & $\begin{array}{l}\text { Education and } \\
\text { practice }\end{array}$ & Low & $\begin{array}{l}\text { Provider } \\
\text { and patient }\end{array}$ & No \\
\hline Bieber $2008^{33}$ & RCT* & 2.55 & $\begin{array}{l}\text { Shared decision- } \\
\text { making }\end{array}$ & $\begin{array}{l}\text { Education and } \\
\text { practice }\end{array}$ & Moderate & $\begin{array}{l}\text { Provider } \\
\text { and patient }\end{array}$ & Yes \\
\hline Bieber $2006^{34}$ & $\mathrm{RCT}^{*}$ & 2.82 & $\begin{array}{l}\text { Shared decision- } \\
\text { making }\end{array}$ & $\begin{array}{l}\text { Education and } \\
\text { practice }\end{array}$ & Moderate & $\begin{array}{l}\text { Provider } \\
\text { and patient }\end{array}$ & Yes \\
\hline Bittner $2016^{35}$ & $\begin{array}{l}\text { Controlled } \\
\text { observational* }\end{array}$ & 2.73 & $\begin{array}{l}\text { Specific } \\
\text { communication } \\
\text { technique }\end{array}$ & Practice & Low & Provider & No \\
\hline Blatt $2010^{36}$ & $\mathrm{RCT}^{*}$ & 2.73 & $\begin{array}{l}\text { Specific } \\
\text { communication } \\
\text { technique }\end{array}$ & Instructions & $\begin{array}{l}\text { Not } \\
\text { reported }\end{array}$ & Provider & No \\
\hline Blödt $2016^{37}$ & $\mathrm{RCT}^{*}$ & 2.55 & $\begin{array}{l}\text { Communication } \\
\text { skills }\end{array}$ & $\begin{array}{l}\text { Education and } \\
\text { practice }\end{array}$ & Moderate & Provider & Yes \\
\hline Boissy $2016^{38}$ & $\begin{array}{l}\text { Controlled } \\
\text { observational }\end{array}$ & 2.33 & Relationship & $\begin{array}{l}\text { Education and } \\
\text { practice }\end{array}$ & Low & Provider & Yes \\
\hline Briel 2006(100) & RCT & 2.73 & Patient-centered care & $\begin{array}{l}\text { Education and } \\
\text { practice }\end{array}$ & Low & Provider & Yes \\
\hline Brock $2011^{40}$ & RCT* & 2.45 & $\begin{array}{l}\text { Specific } \\
\text { communication } \\
\text { technique }\end{array}$ & $\begin{array}{l}\text { Education, } \\
\text { practice, and tool }\end{array}$ & Low & Provider & No \\
\hline Brown $1999^{41}$ & $\mathrm{RCT}^{*}$ & 2.67 & Patient-centered care & $\begin{array}{l}\text { Education and } \\
\text { practice }\end{array}$ & Moderate & Provider & No \\
\hline Brown $2000^{42}$ & $\begin{array}{l}\text { Controlled } \\
\text { observational* }\end{array}$ & 2.00 & $\begin{array}{l}\text { Communication } \\
\text { skills }\end{array}$ & $\begin{array}{l}\text { Education and } \\
\text { tool }\end{array}$ & Moderate & Provider & No \\
\hline Cals 2011(101) & $\mathrm{RCT}$ & 2.64 & $\begin{array}{l}\text { Communication } \\
\text { skills }\end{array}$ & $\begin{array}{l}\text { Education, } \\
\text { practice, and tool }\end{array}$ & Low & Provider & Yes \\
\hline Cals 2013(102) & RCT & 2.82 & $\begin{array}{l}\text { Communication } \\
\text { skills }\end{array}$ & $\begin{array}{l}\text { Education, } \\
\text { practice, and tool }\end{array}$ & Low & Provider & Yes \\
\hline Cooper $2013^{45}$ & $\mathrm{RCT}^{*}$ & 2.55 & Patient-centered care & $\begin{array}{l}\text { Education and } \\
\text { practice }\end{array}$ & Low & $\begin{array}{l}\text { Provider } \\
\text { and patient }\end{array}$ & No \\
\hline Cooper, $2011^{46}$ & $\mathrm{RCT}^{*}$ & 2.36 & Patient-centered care & $\begin{array}{l}\text { Education and } \\
\text { practice }\end{array}$ & $\begin{array}{l}\text { Not } \\
\text { reported }\end{array}$ & $\begin{array}{l}\text { Provider } \\
\text { and patient }\end{array}$ & Yes \\
\hline Curtis $2013^{47}$ & $\mathrm{RCT}$ & 2.64 & $\begin{array}{l}\text { Specific } \\
\text { communication } \\
\text { technique }\end{array}$ & $\begin{array}{l}\text { Education, } \\
\text { practice, and tool }\end{array}$ & Moderate & Provider & No \\
\hline Daly $2010^{48}$ & $\begin{array}{l}\text { Controlled } \\
\text { observational* }\end{array}$ & 2.56 & $\begin{array}{l}\text { Specific } \\
\text { communication } \\
\text { technique }\end{array}$ & $\begin{array}{l}\text { Education and } \\
\text { practice }\end{array}$ & $\begin{array}{l}\text { Not } \\
\text { reported }\end{array}$ & $\begin{array}{l}\text { Provider } \\
\text { and patient }\end{array}$ & No \\
\hline Delvaux $2004^{49}$ & $\mathrm{RCT}^{*}$ & 2.82 & $\begin{array}{l}\text { Specific } \\
\text { communication } \\
\text { technique }\end{array}$ & $\begin{array}{l}\text { Education and } \\
\text { practice }\end{array}$ & High & Provider & No \\
\hline DeMaria $2011^{50}$ & RCT & 2.45 & $\begin{array}{l}\text { Psychological/ } \\
\text { therapeutic interview }\end{array}$ & $\begin{array}{l}\text { Education, } \\
\text { practice, and tool }\end{array}$ & Low & Provider & Yes \\
\hline Dunn $2013^{51}$ & RCT & 2.36 & Mindfulness & $\begin{array}{l}\text { Education, } \\
\text { practice, and tool }\end{array}$ & High & Provider & No \\
\hline
\end{tabular}


Table 2. (continued)

\begin{tabular}{|c|c|c|c|c|c|c|c|}
\hline Study & Study design* & $\begin{array}{l}\text { Quality } \\
\text { assessment }\end{array}$ & Content focus & $\begin{array}{l}\text { Intervention } \\
\text { structure }\end{array}$ & Demand & Recipient & $\begin{array}{l}\text { Framework } \\
\text { (yes/no) }\end{array}$ \\
\hline Edgoose $2014^{52}$ & $\mathrm{RCT}$ & 2.36 & Patient-centered care & Instructions & Low & Provider & Yes \\
\hline Edwards $2004^{53}$ & $\mathrm{RCT}^{*}$ & 2.45 & $\begin{array}{l}\text { Shared decision- } \\
\text { making }\end{array}$ & $\begin{array}{l}\text { Education and } \\
\text { practice }\end{array}$ & Low & Provider & No \\
\hline Epstein $2017^{54}$ & $\mathrm{RCT}^{*}$ & 2.64 & Patient-centered care & $\begin{array}{l}\text { Education and } \\
\text { practice }\end{array}$ & Moderate & $\begin{array}{l}\text { Provider } \\
\text { and patient }\end{array}$ & Yes \\
\hline Finnema $2005^{55}$ & $\mathrm{RCT}$ & 2.64 & $\begin{array}{l}\text { Specific } \\
\text { communication } \\
\text { technique }\end{array}$ & $\begin{array}{l}\text { Education and } \\
\text { practice }\end{array}$ & High & Provider & Yes \\
\hline Fujimori $2014^{56}$ & $\mathrm{RCT}^{*}$ & 2.64 & $\begin{array}{l}\text { Communication } \\
\text { skills }\end{array}$ & $\begin{array}{l}\text { Education and } \\
\text { practice }\end{array}$ & Moderate & Provider & Yes \\
\hline Fukui $2011^{57}$ & RCT* & 2.64 & $\begin{array}{l}\text { Communication } \\
\text { skills }\end{array}$ & $\begin{array}{l}\text { Education, } \\
\text { practice, and tool }\end{array}$ & Moderate & Provider & Yes \\
\hline Fukui $2009^{58}$ & $\mathrm{RCT}^{*}$ & 3.00 & $\begin{array}{l}\text { Communication } \\
\text { skills }\end{array}$ & $\begin{array}{l}\text { Education, } \\
\text { practice, and tool }\end{array}$ & High & Provider & Yes \\
\hline $\begin{array}{l}\text { Grepmair } \\
2007^{59}\end{array}$ & $\mathrm{RCT}$ & 2.82 & Mindfulness & Practice & High & Provider & No \\
\hline Griffey $2015^{60}$ & RCT & 1.82 & Health literacy & $\begin{array}{l}\text { Education and } \\
\text { practice }\end{array}$ & $\begin{array}{l}\text { Not } \\
\text { reported }\end{array}$ & Provider & No \\
\hline Harmsen $2005^{61}$ & RCT & 2.45 & $\begin{array}{l}\text { Specific } \\
\text { communication } \\
\text { technique }\end{array}$ & $\begin{array}{l}\text { Education and } \\
\text { practice }\end{array}$ & Moderate & $\begin{array}{l}\text { Provider } \\
\text { and patient }\end{array}$ & Yes \\
\hline Haskard $2008^{62}$ & RCT* & 2.55 & $\begin{array}{l}\text { Communication } \\
\text { skills }\end{array}$ & $\begin{array}{l}\text { Education, } \\
\text { practice, and tool }\end{array}$ & Moderate & $\begin{array}{l}\text { Provider } \\
\text { and patient }\end{array}$ & No \\
\hline Heritage $2007^{63}$ & $\begin{array}{l}\text { Controlled } \\
\text { observational* }\end{array}$ & 2.64 & $\begin{array}{l}\text { Specific } \\
\text { communication } \\
\text { technique }\end{array}$ & $\begin{array}{l}\text { Education, } \\
\text { instruction, and } \\
\text { tool }\end{array}$ & Low & Provider & No \\
\hline Hietanen $2007^{64}$ & $\mathrm{RCT}$ & 2.73 & $\begin{array}{l}\text { Communication } \\
\text { skills }\end{array}$ & $\begin{array}{l}\text { Education and } \\
\text { practice }\end{array}$ & Moderate & Provider & No \\
\hline Jaffray $2014^{65}$ & RCT & 2.45 & $\begin{array}{l}\text { Motivational } \\
\text { interviewing }\end{array}$ & $\begin{array}{l}\text { Education and } \\
\text { practice }\end{array}$ & Moderate & Provider & No \\
\hline Johnson $2008^{66}$ & $\mathrm{RCT}^{*}$ & 2.64 & $\begin{array}{l}\text { Communication } \\
\text { skills }\end{array}$ & Instructions & Low & Provider & No \\
\hline Jones $2016^{67}$ & RCT & 2.55 & $\begin{array}{l}\text { Motivational } \\
\text { interviewing }\end{array}$ & Education & Moderate & $\begin{array}{l}\text { Provider } \\
\text { and patient }\end{array}$ & Yes \\
\hline $\operatorname{Kim} 2012^{68}$ & RCT* & 2.45 & $\begin{array}{l}\text { Motivational } \\
\text { interviewing }\end{array}$ & $\begin{array}{l}\text { Education, } \\
\text { instruction, and } \\
\text { tool }\end{array}$ & $\begin{array}{l}\text { Not } \\
\text { reported }\end{array}$ & Provider & Yes \\
\hline $\begin{array}{l}\text { Kinmonth } \\
1998^{69}\end{array}$ & RCT & 2.36 & Patient-centered care & $\begin{array}{l}\text { Education, } \\
\text { practice, and tool }\end{array}$ & Moderate & $\begin{array}{l}\text { Provider } \\
\text { and patient }\end{array}$ & No \\
\hline $\begin{array}{l}\text { Langewitz } \\
1998^{70}\end{array}$ & $\mathrm{RCT}^{*}$ & 2.64 & Patient-centered care & $\begin{array}{l}\text { Education, } \\
\text { practice, and tool }\end{array}$ & Moderate & Provider & Yes \\
\hline Little $2015^{71}$ & RCT & 2.45 & $\begin{array}{l}\text { Communication } \\
\text { skills }\end{array}$ & $\begin{array}{l}\text { Education and } \\
\text { practice }\end{array}$ & Low & Provider & Yes \\
\hline Loh $2007^{72}$ & $\mathrm{RCT}$ & 2.73 & $\begin{array}{l}\text { Shared decision- } \\
\text { making }\end{array}$ & $\begin{array}{l}\text { Education, } \\
\text { practice, and tool }\end{array}$ & High & $\begin{array}{l}\text { Provider } \\
\text { and patient }\end{array}$ & Yes \\
\hline Lonsdale $2017^{73}$ & RCT & 3.00 & $\begin{array}{l}\text { Communication } \\
\text { skills }\end{array}$ & Education & Moderate & Provider & Yes \\
\hline Luo $2007^{74}$ & RCT* & 2.73 & Relationship & $\begin{array}{l}\text { Education and } \\
\text { practice }\end{array}$ & $\begin{array}{l}\text { Not } \\
\text { reported }\end{array}$ & Provider & Yes \\
\hline $\begin{array}{l}\text { Maatouk- } \\
\text { Bürmann } \\
2016^{75}\end{array}$ & RCT* & 2.50 & $\begin{array}{l}\text { Communication } \\
\text { skills }\end{array}$ & $\begin{array}{l}\text { Education and } \\
\text { practice }\end{array}$ & High & Provider & Yes \\
\hline Manze $2015^{76}$ & RCT & 2.82 & $\begin{array}{l}\text { Communication } \\
\text { skills }\end{array}$ & $\begin{array}{l}\text { Education, } \\
\text { practice, and tool }\end{array}$ & Low & $\begin{array}{l}\text { Provider } \\
\text { and patient }\end{array}$ & Yes \\
\hline Mercer $2016^{77}$ & RCT & 2.64 & Mindfulness & $\begin{array}{l}\text { Education, } \\
\text { practice, and tool }\end{array}$ & High & $\begin{array}{l}\text { Provider } \\
\text { and patient }\end{array}$ & Yes \\
\hline $\begin{array}{l}\text { Merckaert } \\
2015^{78}\end{array}$ & RCT & 2.55 & $\begin{array}{l}\text { Communication } \\
\text { skills }\end{array}$ & $\begin{array}{l}\text { Education and } \\
\text { practice }\end{array}$ & High & Provider & No \\
\hline $\begin{array}{l}\text { Middleton } \\
2006^{79}\end{array}$ & RCT & 2.64 & $\begin{array}{l}\text { Specific } \\
\text { communication } \\
\text { technique }\end{array}$ & $\begin{array}{l}\text { Education, } \\
\text { practice, and tool }\end{array}$ & Low & $\begin{array}{l}\text { Provider } \\
\text { and patient }\end{array}$ & Yes \\
\hline Moral $2015^{80}$ & RCT & 2.42 & $\begin{array}{l}\text { Motivational } \\
\text { interviewing }\end{array}$ & $\begin{array}{l}\text { Education and } \\
\text { practice }\end{array}$ & Moderate & Provider & Yes \\
\hline $\begin{array}{l}\text { Muñoz Alamo } \\
2002^{81}\end{array}$ & RCT & 2.45 & Patient-centered care & $\begin{array}{l}\text { Education and } \\
\text { practice }\end{array}$ & Moderate & Provider & Yes \\
\hline Penner $2013^{82}$ & RCT & 2.36 & Patient-centered care & $\begin{array}{l}\text { Instructions and } \\
\text { tools }\end{array}$ & Low & $\begin{array}{l}\text { Provider } \\
\text { and patient }\end{array}$ & Yes \\
\hline Pill $1998^{83}$ & $\mathrm{RCT}^{*}$ & 2.55 & Patient-centered care & $\begin{array}{l}\text { Education, } \\
\text { practice, and tool }\end{array}$ & Moderate & Provider & Yes \\
\hline Rakel $2011^{84}$ & $\mathrm{RCT}$ & 2.73 & $\begin{array}{l}\text { Specific } \\
\text { communication } \\
\text { technique }\end{array}$ & $\begin{array}{l}\text { Education, } \\
\text { practice, and tool }\end{array}$ & $\begin{array}{l}\text { Not } \\
\text { reported }\end{array}$ & $\begin{array}{l}\text { Provider } \\
\text { and patient }\end{array}$ & Yes \\
\hline Rask $2009^{85}$ & RCT & 2.45 & & & High & Provider & No \\
\hline
\end{tabular}


Table 3 Impact on the Quadruple Aim by Focus of the Intervention

\begin{tabular}{|c|c|c|c|c|c|}
\hline \multirow[t]{2}{*}{ Content focus } & \multicolumn{5}{|c|}{ Studies with at least one positive outcome relevant to the quadruple aim } \\
\hline & $\begin{array}{l}\text { Health } \\
\text { outcomes }\end{array}$ & Patient experience & $\begin{array}{l}\text { Provider } \\
\text { experience }\end{array}$ & $\begin{array}{l}\text { Cost-related } \\
\text { outcomes }\end{array}$ & $\begin{array}{l}\text { No significant improvement in } \\
\text { outcomes for all investigated domains }\end{array}$ \\
\hline $\begin{array}{l}\text { Motivational } \\
\text { interviewing }\end{array}$ & $67,80,90$ & 67,68 & 87 & & 65 \\
\hline Health literacy & 26 & 21,60 & 21 & & \\
\hline Relationship & 74 & 38 & & & 91 \\
\hline Patient-centered care & $28,69,81,82$ & $45,46,54,69,82,83,93$ & $46,52,70$ & & $39,41,92$ \\
\hline Communication skills & $44,56-58,73$ & $\begin{array}{l}42,56,57,62,64,66,71, \\
73,78,86,89\end{array}$ & $\begin{array}{l}42,56,58,62,75 \\
78,86,89\end{array}$ & 23 & $25,30,37,43,76,85$ \\
\hline Shared decision-making & & $33,34,53,72,88$ & & & \\
\hline $\begin{array}{l}\text { Specific communication } \\
\text { technique }\end{array}$ & $22,31,55$ & $27,35,36,49,63,79,84$ & $34,40,49,55,79$ & & $29,32,47,48,61$ \\
\hline Psychological/ & & 24,50 & & & \\
\hline $\begin{array}{l}\text { therapeutic interview } \\
\text { Mindfulness }\end{array}$ & 59,77 & 51 & 51 & 77 & \\
\hline
\end{tabular}

*Detailed outcomes for each quadruple aim domain (including nonsignificant findings) are presented in Tables 15 and 16 in the Appendix

(e.g., instruction to sit vs. stand during the visit), two interventions (3\%) encompassed only education, and one was limited to a tool (Table 9 in the Appendix). (i.e., a one-page feedback form to aid in stimulating conversation with patients). Only 22 (30\%) interventions had a dyadic (e.g., patient-provider) approach; the others were provider-focused only (Table 10 in the Appendix). Thirty-four studies (47\%) used methods to directly observe communication; 28 of these found a positive association between intervention efforts and a quadruple aim outcome. Table 2 presents intervention characteristics for each study, and Table 5 in the Appendix presents intervention setting and participant demographic information for each study.

\section{Impact on the Quadruple Aim Outcomes}

Table 3 presents the studies that had at least one positive outcome relevant to the quadruple aim, by content focus.
Below, we describe an overview of findings for each quadruple aim domain. Refer to Tables 15 and 16 in the Appendix for the list of health and cost outcomes and patient and provider experience outcomes, respectively.

Health Outcomes. Among 38 (52\%) studies that included health measures, moderate-demand interventions that focused on a specific communication technique ${ }^{22,31,47}$ had a positive impact on several health outcomes, including physical function, obesity control, and mental health. Several interventions that comprised a practice alone, or combined education and practice coupled with a tool, also improved health outcomes (Table 11 in the Appendix presents health outcomes by study). These results suggest that moderate- to high-demand interventions focused on a specific communication technique, partnered with an education-practice-tool intervention, may offer an effective mechanism to influence health outcomes. As an example, Bellón et al. evaluated a communication technique

Table 2. (continued)

\begin{tabular}{|c|c|c|c|c|c|c|c|}
\hline Study & Study design* & $\begin{array}{l}\text { Quality } \\
\text { assessment }\end{array}$ & Content focus & $\begin{array}{l}\text { Intervention } \\
\text { structure }\end{array}$ & Demand & Recipient & $\begin{array}{l}\text { Framework } \\
\text { (yes/no) }\end{array}$ \\
\hline & & & $\begin{array}{l}\text { Communication } \\
\text { skills }\end{array}$ & $\begin{array}{l}\text { Education and } \\
\text { practice }\end{array}$ & & & \\
\hline Roter $1998^{86}$ & $\begin{array}{l}\text { Controlled } \\
\text { observational } *\end{array}$ & 2.50 & $\begin{array}{l}\text { Communication } \\
\text { skills }\end{array}$ & $\begin{array}{l}\text { Education, } \\
\text { practice, and tool }\end{array}$ & Moderate & Provider & No \\
\hline Smith $1998^{87}$ & $\mathrm{RCT}^{*}$ & 2.82 & $\begin{array}{l}\text { Motivational } \\
\text { interviewing }\end{array}$ & $\begin{array}{l}\text { Education and } \\
\text { practice }\end{array}$ & Moderate & Provider & Yes \\
\hline Song $2015^{88}$ & $\mathrm{RCT}^{*}$ & 2.64 & $\begin{array}{l}\text { Shared decision- } \\
\text { making }\end{array}$ & $\begin{array}{l}\text { Education and } \\
\text { practice }\end{array}$ & Moderate & Provider & Yes \\
\hline Stewart $2007^{89}$ & $\mathrm{RCT}^{*}$ & 2.27 & $\begin{array}{l}\text { Communication } \\
\text { skills }\end{array}$ & $\begin{array}{l}\text { Education and } \\
\text { practice }\end{array}$ & Low & Provider & Yes \\
\hline Swanson $1999^{90}$ & $\mathrm{RCT}$ & 2.42 & $\begin{array}{l}\text { Motivational } \\
\text { interviewing }\end{array}$ & $\begin{array}{l}\text { Education, } \\
\text { practice, and tool }\end{array}$ & Low & Provider & Yes \\
\hline Thom $1999^{91}$ & RCT & 2.45 & Relationship & $\begin{array}{l}\text { Education and } \\
\text { practice }\end{array}$ & Low & Provider & Yes \\
\hline $\begin{array}{l}\text { Wolf } 2008 \\
(\text { JNCQ })^{92}\end{array}$ & RCT & 2.27 & Patient-centered care & $\begin{array}{l}\text { Education and } \\
\text { practice }\end{array}$ & Moderate & $\begin{array}{l}\text { Provider } \\
\text { and patient }\end{array}$ & No \\
\hline $\begin{array}{l}\text { Wolf } 2008 \\
\text { (JONA) }^{93}\end{array}$ & RCT & 2.36 & Patient-centered care & $\begin{array}{l}\text { Education and } \\
\text { practice }\end{array}$ & Moderate & $\begin{array}{l}\text { Provider } \\
\text { and patient }\end{array}$ & Yes \\
\hline
\end{tabular}

*Indicates that direct observation was used to evaluate effects of the intervention 
intervention that encourages general practitioners to select from a list of seven possible hypotheses for why a patient is a frequent attender (i.e., biological, psychological, social, family, cultural, administrative-organizational, or related to doctor-patient relationship), and then share their selection with colleagues along with a plan to address these issues. The intervention training involved an educational component (3-day workshop) coupled with skills-based practice and a pocket guide tool and was found to reduce the number of consultations among frequent attenders by $61.3 \%$ at 1 year. ${ }^{31}$

Patient Experience. Most studies (74\%, 49 RCTs and 5 controlled observational studies) evaluated patient experience outcomes; there was marked variation in the intervention focus, structure, and demand associated with positive impact on patient experience (Table 12 in the Appendix presents patient experience outcomes by study). Among the 38 studies that reported a positive patient experience outcome, $61 \%$ used provider-focused interventions. This suggests that interventions may improve patient perceptions of their care without requiring active participation by the patient in the intervention (although there are likely bidirectional factors such that changes in the provider's communication behavior affect the patient's communication behavior as well). Among interventions with a positive impact on patient experience (e.g., satisfaction, comprehension), several also demonstrated a positive impact on health outcomes (e.g., depression, adherence). In some cases, interventions that positively influenced patient experience also had a positive impact on provider experience and cost. As an example, Brock et al. evaluated a low-demand specific communication technique that prompted providers to (1) familiarize the patient with the "establishing focus" process; (2) ask the patient to list concerns; (3) make space for pressing patient stories early on; (4) avoid prematurely initiating diagnostic sequences or patient storytelling before a full agenda is set; (5) ask patient to prioritize concerns; (6) negotiate priorities, when necessary; and (7) seek confirmation and commitment from the patient. A brief education (2-h group training session, handbook, videotape demonstration, group discussion) and practice (role-play, coaching) coupled with a tool (video and cue card describing "establishing focus" protocol) reduced the number of concerns raised by patients near the end of the visit (1.14 to 0.83 concerns per patient) without influencing visit length (a proxy for cost of care). ${ }^{40}$

Cost of Care. Nineteen (26\%) studies examined cost as an outcome or evaluated outcomes that could potentially generate savings (e.g., imaging efficiency, prescription rates). Among these, only three studies suggested savings could be attributed to the intervention. ${ }^{23,43,77}$ Among the remaining 16 studies, $10(53 \%)$ found no significant differences in cost or cost- related outcomes, and $6(32 \%)$ reported at least one instance of increased costs or a cost-related outcome. In one costeffectiveness study, Mercer et al. used a provider- and patientfocused intervention that included education about the CARE Plus program, practice (group-based support and training), and a clinic-based tool to aid providers in identifying patient concerns and priorities, promote patient self-management, and ensure agreement in care plan. Patients also received a mindfulnessbased stress management $\mathrm{CD}$, a cognitive behavioral therapy self-help booklet, and written material about the intervention. Although the intervention was high demand in terms of the time investment for clinicians, it resulted in improved patient wellbeing (as measured by W-BQ12) and improvement in qualityadjusted life years of 0.076 (95\% CI $0.028-0.124$ ) over the 12 month trial, resulting in a cost-effectiveness ratio of $£ 12,224$ per quality-adjusted life year gained. ${ }^{77}$

Eleven studies (10 RCTs and 1 controlled observational study) measured clinic visit length, which has the potential to influence cost (Table 13 in the Appendix). Only four of those studies found that visits were significantly longer in the intervention group compared to the control group (two studies utilized the same data set, which found longer visits for nurse interviews but not physician interviews). ${ }^{57}, 58,75,84$ These studies also identified significant improvements in areas of patient health (i.e., quality of life), patient experience (i.e., satisfaction), and provider experience (i.e., communication ability). In five studies, the intervention did not significantly influence visit time, but there was a positive impact on patient satisfaction, ${ }^{50,57,72}$ perceived patient-centeredness, ${ }^{40,72}$ and number of unmet concerns among patients. ${ }^{9}$ These findings suggest that interpersonal interventions can have positive benefits on patient experience with minimal impact on visit length.

Provider Experience. As with patient experience, among studies examining provider experience $(n=27)$, we observed variation across focus, structure, demand, and recipient of the intervention (Table 14 in the Appendix presents provider experience outcomes by study). One of the most common types of interventions that positively influenced provider experience was clinician training using a general communication content focus $(n=11)$, which was associated with improvements in communication, satisfaction, and perceived provider friendliness. Many of the studies examining provider experience did not describe training time or duration. Collectively, results suggest that interpersonal interventions may improve provider communication practices, increase their self-efficacy in connecting with the patient, and reduce burnout. For example, Delvaux et al. evaluated a high-demand, provider-focused intervention that involved education (105-h psychological training program) and practice to implement a specific communication skill in the 
oncology setting. The intervention reduced stress among nurses (by an average of 0.2 on a 4-point Nursing Stress Scale), improved nurses' attitudes by 0.2 on the 7-point semantic differential questionnaire, and increased nurse communication skills (Nurses Satisfaction with the Interview Assessment Questionnaire and Patient Satisfaction with the Interview Assessment Questionnaire; 8-item four-point scale) with an average effect size of $0.7 .^{49}$

\section{DISCUSSION}

This systematic review of 73 studies of interpersonal interventions identified several intervention features that may positively influence patient health and patient and provider experience; fewer studies found significant positive effects on cost outcomes. Our review updates and extends previous work by synthesizing interventions according to the widely accepted goals of the quadruple aim (population health, patient experience, cost, and provider experience). The following discussion considers the implications of these findings for clinical practice and health system redesign.

A common misperception is that interpersonal skills are innate or cannot be taught. ${ }^{94}$ Our review confirms the opposite, that quite a number of interventions can be employed to improve patient-provider interactions and downstream outcomes. Encouragingly, some of these effective interventions are also relatively efficient. Several low- to moderate-demand interventions had positive impacts on a range of quadruple aim-related outcomes, including health outcomes (e.g., physical function and adherence), patient experience (e.g., satisfaction, involvement in care, and comprehension), and provider experience (e.g., reduced depersonalization and emotional exhaustion) (Table 7 in the Appendix).

What are key components of interpersonal interventions that may foster human connection in medical care? The reviewed studies provide some possible clues. Several of the studies with a positive impact on patient or provider experience focused on communication skills $(18 \%)$ or a specific communication technique (14\%), such as eliciting patient priorities or teaching a specific phrase to elicit unaddressed concerns. ${ }^{66}$ However, interventions focusing on content areas ranging from mindfulness techniques to shared decision-making also showed promise Table 2 . Many interventions employed multiple modalities that commonly included education and practice $(48 \%)$ or education and practice accompanied by a tool (33\%); results regarding the effectiveness of using a combination of training modalities are mixed (Table 9 in the Appendix). Finally, connection is bidirectional, yet in most of the reviewed studies, the intervention focused solely on changing provider behavior and many of these were effective (Table 10 in the Appendix). At present, there is insufficient evidence to conclusively compare the merits of provider-only versus provider-patient dyad interventions. This may be a useful focus for future research.

The decision to employ interpersonal skill interventions will depend greatly on their complexity and cost. Unfortunately, these structural aspects of the interventions were not always described in sufficient detail. For example, several studies failed to report intervention training time and only 11 studies measured visit time as an outcome. Among the studies that did report this information, however, most found that the interventions improved patient and provider experience without significantly increasing visit time (Table 13 in the Appendix).

Downstream cost savings would be another argument for adopting interpersonal interventions, and 19 studies took this into consideration. Only three studies found potential cost savings associated with an interpersonal intervention. ${ }^{23,43,77}$ These results suggest that it should not be assumed that enhancing interpersonal communication will increase efficiency or reduce costs. Given the relative paucity of evidence, however, continuing to assess cost and cost-related outcomes would elevate the value of future studies of interpersonal interventions.

Our approach has several limitations. Like all systematic reviews, our evidence synthesis is subject to both publication and selection bias. Given the breadth of this topic, we may have missed relevant studies that did not include a required MeSH or "text word" search term from both concept 1 (interpersonal intervention) and concept 2 (quadruple aim outcome) of our search strategy (Table 4 in the Appendix). In addition, by restricting our review to RCTs and controlled observational studies, our findings represent only a portion of relevant research pertaining to interpersonal interventions and their implementation. ${ }^{94} \mathrm{We}$ narrowed our focus in an effort to synthesize the evidence across studies with similar designs; however, future research could gain valuable insight from adaptive and pragmatic trials, pre-post studies, and qualitative research. Moreover, future research might benefit from taking a more direct approach to examine patient-provider interactions themselves, at varying levels of interpersonal competency, and to evaluate whether better interactions are associated with improved quadruple aim outcomes. ${ }^{4}, 89$ Additional research is also needed to synthesize the evidence around interpersonal interactions with caregivers and interactions between patients and allied health professionals. Our review does not account for team or clinical microsystem impacts that may affect interpersonal interactions in the patient-provider encounter. ${ }^{95}$

Another limitation of our research is the inability to conduct a meta-analysis of the data due to the 
heterogeneity in study designs and outcomes. Variability among studies further complicates the synthesis of results. The negative findings across studies may be attributed to beta error, due to inadequate sample size, which should be considered when interpreting the results. We found that several studies express overlapping aims that could undermine validity and generalizability of findings. Similarly, because of the breadth of intervention approaches and studies that lacked sufficient and clear descriptions, we are unable to offer a detailed account of each intervention in the synthesis of studies, but instead provide a resource for locating interventions and guiding frameworks that are of interest based on intervention content and impact across a range of outcomes (Table 2). Furthermore, with complex social interventions, it is challenging to assess sustained impact, which presents another consideration in the interpretation of our findings. ${ }^{96}$ Finally, our search strategy was limited to English-language studies, which may have excluded relevant interventions (Table 4 in the Appendix).

In summary, this review provides a comprehensive overview of the evidence regarding the impact of patient-provider interpersonal interventions on quadruple aim outcomes. Our review of interpersonal interventions that met inclusion criteria suggests that interventions that require only minimal to moderate demands may have the potential to improve patient experience, provider experience, and features of patient health. Limited evidence suggests that these interventions may achieve these improvements without prolonging visit time. Studies examining downstream cost savings showed minimal benefits, and further exploration of this outcome is needed. Human connection is at the heart of clinical care, and our findings suggest that clinicians, managers, and policymakers should consider interpersonal interventions not only for their inherent value, but because of the potential for impact across the quadruple aim.

Acknowledgments: We would like to acknowledge Ted Miclau, Laura Jacobson, Shreyas Bharadwaj, and Isabella Romero for their assistance in data analysis and synthesis. We would also like to acknowledge Amrapali Maitra, Sheila Lahijani, and Lars Osterberg for their insightful contributions to this research study.

Corresponding Author: Marie C. Haverfield, PhD; Stanford University Center for Primary Care and Outcomes Research (PCOR) and Center for Health Research and Policy (CHRP), Stanford, CA, USA (e-mail: marie.haverfield@sjsu.edu).

Funding Information This study was supported by a grant from the Gordon and Betty Moore Foundation (\#6382, Zulman \& Verghese, PIs) and a grant from the Arthur Vining Davis Foundation. Drs. Haverfield and Schwartz are supported by VA Palo Alto Center for Innovation to Implementation (Ci2i) HSR\&D postdoctoral fellowship.

\section{Compliance with Ethical Standards:}

Conflict of Interest: There are no conflicts to disclose.

\section{REFERENCES}

1. Verghese A, Charlton B, Cotter B, Kugler J. A history of physical examination texts and the conception of bedside diagnosis. Trans Am Clin Climatolo Assoc. 2011;122:290-311.

2. Chi J, Verghese A. Improving communication with patients: learning by doing. JAMA. 2013;310(21):2257-8.

3. Epstein RM, Franks P, Fiscella K, Shields CG, Meldrum SC, Kravitz RL, et al. Measuring patient-centered communication in patientphysician consultations: theoretical and practical issues. Soc Sci Med. 2005;61(7):1516-28.

4. Zolnierek KB, Dimatteo MR. Physician communication and patient adherence to treatment: a meta-analysis. Med Care. 2009;47(8):826-34.

5. Verghese A, Charlton B, Kassirer JP, Ramsey M, Ioannidis JP. Inadequacies of physical examination as a cause of medical errors and adverse events: a collection of vignettes. Am J Med. 2015;128(12):13224.e3.

6. Passalacqua SA, Segrin C. The effect of resident physician stress, burnout, and empathy on patient-centered communication during the long-call shift. Health Commun 2012;27(5):449-56.

7. Rodin G, Zimmermann C, Mayer C, Howell D, Katz M, Sussman J, et al. Clinician-patient communication: evidence-based recommendations to guide practice in cancer. Curr Oncol. 2009;16(6):42-9.

8. Hoerger M, Epstein RM, Winters PC, Fiscella $\mathbf{K}$, Duberstein PR, Gramling R, et al. Values and options in cancer care (VOICE): study design and rationale for a patient-centered communication and decisionmaking intervention for physicians, patients with advanced cancer, and their caregivers. BMC Cancer 2013;13:188.

9. Heritage J, Robinson JD. The structure of patients' presenting concerns: physicians' opening questions. Health Commun. 2006;19(2):89102.

10. Mead N BP. Patient-centeredness: a conceptual framework and review of the empirical literature. Soc Sci Med 2000;51(7):1087-110.

11. Griffin SJ, Kinmonth AL, Veltman MW, Gillard S, Grant J, Stewart M. Effect on health-related outcomes of interventions to alter the interaction between patients and practitioners: a systematic review of trials. Ann Fam Med 2004;2(6):595-608.

12. Rodin G, Mackay JA, Zimmermann C, Mayer C, Howell D, Katz M, et al. Clinician-patient communication: a systematic review. Support Care Cancer 2009;17(6):627-44.

13. De Vries AM, de Roten Y, Meystre C, Passchier J, Despland JN, Stiefel F. Clinician characteristics, communication, and patient outcome in oncology: a systematic review. Psychooncology. 2014;23(4):375-81.

14. Kelley JM, Kraft-Todd G, Schapira L, Kossowsky J, Riess H. The influence of the patient-clinician relationship on healthcare outcomes: a systematic review and meta-analysis of randomized controlled trials. PloS One 2014;9(4):e94207.

15. Bodenheimer T, Sinsky C. From triple to quadruple aim: care of the patient requires care of the provider. Ann Fam Med 2014;12(6):573-6.

16. Berwick DM, Nolan TW, Whittington $\mathbf{J}$. The triple aim: care, health, and cost. Health Aff (Milwood)2008;27(3):759-69.

17. Wood JT. Interpersonal Communication: Everyday Encounters. 7th ed. Boston: Cengage Learning; 2013.

18. Cochrane Effective Practice and Organisation of Care (EPOC). EPOC Resources for review authors, 2017. Available at: https://www.epoc. cochrane.org/epoc-resources-review-authors

19. Phillips B BC, Sackett D, Badenoch D, Straus S, Haynes B, Dawes M. Oxford Centre for Evidence-Based Medicine-Level of Evidence [Internet]. Oxford: Oxford Centre for Evidence-Based Medicine; 19981998 Nov [updated 2009 Mar; cited 2018 Mar 29].

20. Rao JK, Anderson LA, Inui TS, Frankel RM. Communication interventions make a difference in conversations between physicians and patients: a systematic review of the evidence. Med Care 2007;45(4):340 9.

21. Aboumatar HJ, Carson KA, Beach MC, Roter DL, Cooper LA. The impact of health literacy on desire for participation in healthcare, medical visit communication, and patient reported outcomes among patients with hypertension. J Gen Intern Med 2013;28(11):1469-76.

22. Aiarzaguena JM, Grandes G, Gaminde I, Salazar A, Sanchez A, Arino J. A randomized controlled clinical trial of a psychosocial and communication intervention carried out by GPs for patients with medically unexplained symptoms. Psychol Med 2007;37(2):283-94. 
23. Ajam AA, Nguyen XV, Kelly RA, Ladapo JA, Lang EV. Effects of interpersonal skills training on MRI operations in a saturated market: a randomized trial. J Am Coll Radiol 2017;14(7):963-70.

24. Akturan S, Kaya CA, Unalan PC, Akman M. The effect of the BATHE interview technique on the empowerment of diabetic patients in primary care: a cluster randomised controlled study. Prim Care Diabetes 2017;11(2):154-61.

25. Alder J, Christen R, Zemp E, Bitzer J. Communication skills training in obstetrics and gynaecology: whom should we train? A randomized controlled trial. Arch Gynecol Obstet 2007;276(6):605-12.

26. Altiner A, Brockmann S, Sielk M, Wilm S, Wegscheider K, Abholz HH. Reducing antibiotic prescriptions for acute cough by motivating GPs to change their attitudes to communication and empowering patients: a cluster-randomized intervention study. J Antimicrob Chemother 2007;60(3):638-44.

27. Au DH, Udris EM, Engelberg RA, Diehr PH, Bryson CL, Reinke LF, et al. A randomized trial to improve communication about end-of-life care among patients with COPD. Chest. 2012;141(3):726-35.

28. Aubin-Auger I, Laouenan C, Le Bel J, Mercier A, Baruch D, Lebeau JP, et al. Efficacy of communication skills training on colorectal cancer screening by GPs: a cluster randomised controlled trial. Eur J Cancer Care (Engl) 2016;25(1):18-26.

29. Bakker IM, Terluin B, van Marwijk HW, van der Windt DA, Rijmen F, van Mechelen W, et al. A cluster-randomised trial evaluating an intervention for patients with stress-related mental disorders and sick leave in primary care. PLoS Clinical Trials. 2007;2(6):e26.

30. Bashour HN, Kanaan M, Kharouf MH, Abdulsalam AA, Tabbaa MA, Cheikha SA. The effect of training doctors in communication skills on women's satisfaction with doctor-woman relationship during labour and delivery: a stepped wedge cluster randomised trial in Damascus. BMJ Open. 2013;3(8)

31. Bellon JA, Rodriguez-Bayon A, de Dios Luna J, Torres-Gonzalez F. Successful GP intervention with frequent attenders in primary care: randomised controlled trial. Br J Gen Pract. 2008;58(550):324-30.

32. Bernhard J, Butow P, Aldridge J, Juraskova I, Ribi $\mathbf{K}$, Brown $\mathbf{R}$ Communication about standard treatment options and clinical trials: can we teach doctors new skills to improve patient outcomes? Psychooncology. 2012;21(12):1265-74.

33. Bieber C, Muller KG, Blumenstiel K, Hochlehnert A, Wilke S, Hartmann M, et al. A shared decision-making communication training program for physicians treating fibromyalgia patients: effects of a randomized controlled trial. J Psychosom Res 2008;64(1):13-20.

34. Bieber C, Muller KG, Blumenstiel K, Schneider A, Richter A, Wilke S, et al. Long-term effects of a shared decision-making intervention on physician-patient interaction and outcome in fibromyalgia. A qualitative and quantitative 1 year follow-up of a randomized controlled trial. Patient Educ Couns. 2006;63(3):357-66.

35. Bittner A, Bittner J, Jonietz A, Dybowski C, Harendza S. Translating medical documents improves students' communication skills in simulated physician-patient encounters. BMC Med Educ. 2016;16:72.

36. Blatt B, LeLacheur SF, Galinsky AD, Simmens SJ, Greenberg L. Does perspective-taking increase patient satisfaction in medical encounters? Acad Med. 2010;85(9): 1445-52.

37. Blodt S, Mittring N, Schutzler L, Fischer F, Holmberg C, Horneber M, et al. A consultation training program for physicians for communication about complementary medicine with breast cancer patients: a prospective, multi-center, cluster-randomized, mixed-method pilot study. BMC Cancer. 2016; 16(1):843

38. Boissy A, Windover AK, Bokar D, Karafa M, Neuendorf K, Frankel RM, et al. Communication skills training for physicians improves patient satisfaction. J Gen Intern Med. 2016;31(7):755-61.

39. Briel M, Langewitz W, Tschudi P, Young J, Hugenschmidt C, Bucher HC. Communication training and antibiotic use in acute respiratory tract infections. A cluster randomised controlled trial in general practice. Swiss Medical Wkly. 2006;136(15-16):241-7.

40. Brock DM, Mauksch LB, Witteborn S, Hummel J, Nagasawa P, Robins LS. Effectiveness of intensive physician training in upfront agenda setting. J Gen Intern Med. 2011;26(11):1317-23.

41. Brown JB, Boles M, Mullooly JP, Levinson W. Effect of clinician communication skills training on patient satisfaction. A randomized, controlled trial. Ann Intern Med. 1999;131(11):822-9.
42. Brown LD, de Negri B, Hernandez O, Dominguez L, Sanchack JH, Roter D. An evaluation of the impact of training Honduran health care providers in interpersonal communication. Int $\mathrm{J}$ Qual Health Care. 2000; 12(6):495-501

43. Cals JW, Ament AJ, Hood K, Butler CC, Hopstaken RM, Wassink GF, et al. C-reactive protein point of care testing and physician communication skills training for lower respiratory tract infections in general practice: economic evaluation of a cluster randomized trial. J Eval Clin Pract. 2011;17(6):1059-69.

44. Cals JW, de Bock L, Beckers PJ, Francis NA, Hopstaken RM, Hood K, et al. Enhanced communication skills and C-reactive protein point-ofcare testing for respiratory tract infection: 3.5-year follow-up of a cluster randomized trial. Ann Fam Med. 2013;11(2):157-64.

45. Cooper LA, Ghods Dinoso BK, Ford DE, Roter DL, Primm AB, Larson SM, et al. Comparative effectiveness of standard versus patient-centered collaborative care interventions for depression among African Americans in primary care settings: the BRIDGE Study. Health Serv Res. 2013;48(1):150-74.

46. Cooper LA, Roter DL, Carson KA, Bone LR, Larson SM, Miller ER, 3rd, et al. A randomized trial to improve patient-centered care and hypertension control in underserved primary care patients. J Gen Intern Med. 2011:26(11):1297-304.

47. Curtis JR, Back AL, Ford DW, Downey L, Shannon SE, Doorenbos AZ, et al. Effect of communication skills training for residents and nurse practitioners on quality of communication with patients with serious illness: a randomized trial. JAMA. 2013;310(21):2271-81.

48. Daly BJ, Douglas SL, O'Toole E, Gordon NH, Hejal R, Peerless J, et al. Effectiveness trial of an intensive communication structure for families of long-stay ICU patients. Chest. 2010;138(6):1340-8.

49. Delvaux N, Razavi D, Marchal S, Bredart A, Farvacques C, Slachmuylder JL. Effects of a 105 hours psychological training program on attitudes, communication skills and occupational stress in oncology: a randomised study. Br J Cancer. 2004;90(1):106-14.

50. DeMaria S, Jr., DeMaria AP, Silvay G, Flynn BC. Use of the BATHE method in the preanesthetic clinic visit. Anesth Analg. 2011;113(5):10206.

51. Dunn R, Callahan JL, Swift JK, Ivanovic M. Effects of pre-session centering for therapists on session presence and effectiveness. Psychother Res. 2013;23(1):78-85.

52. Edgoose $\boldsymbol{J Y}$, Regner CJ, Zakletskaia LI. BREATHE OUT: a randomized controlled trial of a structured intervention to improve clinician satisfaction with "difficult" visits. J Am Board Fam Med. 2015;28(1):13-20.

53. Edwards A, Elwyn G, Hood K, Atwell C, Robling M, Houston H, et al. Patient-based outcome results from a cluster randomized trial of shared decision making skill development and use of risk communication aids in general practice. Fam Pract. 2004;21(4):347-54.

54. Epstein RM, Duberstein PR, Fenton JJ, Fiscella K, Hoerger M, Tancredi DJ, et al. Effect of a patient-centered communication intervention on oncologist-patient communication, quality of life, and health care utilization in advanced cancer: the VOICE randomized clinical trial. JAMA Oncol. 2017;3(1):92-100.

55. Finnema E, Droes RM, Ettema T, Ooms M, Ader H, Ribbe M, et al. The effect of integrated emotion-oriented care versus usual care on elderly persons with dementia in the nursing home and on nursing assistants: a randomized clinical trial. Int J Geriatr Psychiatry. 2005;20(4):330-43.

56. Fujimori M, Shirai Y, Asai M, Kubota K, Katsumata N, Uchitomi Y. Effect of communication skills training program for oncologists based on patient preferences for communication when receiving bad news: a randomized controlled trial. J Clin Oncol 2014;32(20):2166-72.

57. Fukui S, Ogawa $\mathbf{K}$, Yamagishi A. Effectiveness of communication skills training of nurses on the quality of life and satisfaction with healthcare professionals among newly diagnosed cancer patients: a preliminary study. Psychooncology. 2011;20(12):1285-91.

58. Fukui S, Ogawa K, Ohtsuka M, Fukui N. Effect of communication skills training on nurses' detection of patients' distress and related factors after cancer diagnosis: a randomized study. Psychooncology. 2009; 18(11):1156-64.

59. Grepmair L, Mitterlehner F, Loew T, Bachler E, Rother W, Nickel M. Promoting mindfulness in psychotherapists in training influences the treatment results of their patients: a randomized, double-blind, controlled study. Psychother Psychosom. 2007;76(6):332-8. 
60. Griffey RT, Shin N, Jones S, Aginam N, Gross M, Kinsella Y, et al. The impact of teach-back on comprehension of discharge instructions and satisfaction among emergency patients with limited health literacy: a randomized, controlled study. J Commun Healthc. 2015;8(1):10-21.

61. Harmsen H, Bernsen R, Meeuwesen L, Thomas S, Dorrenboom G, Pinto D, et al. The effect of educational intervention on intercultural communication: results of a randomised controlled trial. Br J Gen Pract. 2005;55(514):343-50.

62. Haskard KB, Williams SL, DiMatteo MR, Rosenthal R, White MK, Goldstein MG. Physician and patient communication training in primary care: effects on participation and satisfaction. Health Psychol 2008;27(5):513-22.

63. Heritage J, Robinson JD, Elliott MN, Beckett M, Wilkes M. Reducing patients' unmet concerns in primary care: the difference one word can make. J Gen Intern Med. 2007;22(10):1429-33.

64. Hietanen PS, Aro AR, Holli KA, Schreck M, Peura A, Joensuu HT. A short communication course for physicians improves the quality of patient information in a clinical trial. Acta Oncol. 2007;46(1):42-8.

65. Jaffray M, Matheson C, Bond CM, Lee AJ, McLernon DJ, Johnstone A, et al. Does training in motivational interviewing for community pharmacists improve outcomes for methadone patients? A cluster randomised controlled trial. Int $J$ Pharm Pract. 2014;22(1):4-12.

66. Johnson RL, Sadosty AT, Weaver AL, Goyal DG. To sit or not to sit? Ann Emerg Med. 2008;51(2):188-93, 93 e1-2.

67. Jones DL, Sued O, Cecchini D, Bofill L, Cook R, Lucas M, et al. Improving adherence to care among "hard to reach" HIV-infected patients in Argentina. AIDS Behav. 2016;20(5):987-97.

68. Kim JH, Park YN, Park EW, Cheong YS, Choi EY. Effects of BATHE interview protocol on patient satisfaction. Korean J Fam Med. 2012;33(6):366-71.

69. Kinmonth AL, Woodcock A, Griffin S, Spiegal N, Campbell MJ. Randomised controlled trial of patient centred care of diabetes in general practice: impact on current wellbeing and future disease risk. The Diabetes Care From Diagnosis Research Team. BMJ. 1998;317(7167):1202-8.

70. Langewitz WA, Eich P, Kiss A, Wossmer B. Improving communication skills-a randomized controlled behaviorally oriented intervention study for residents in internal medicine. Psychosom Med. 1998;60(3):268-76.

71. Little $\mathbf{P}$, White $\mathbf{P}$, Kelly J, Everitt $\mathbf{H}$, Mercer S. Randomised controlled trial of a brief intervention targeting predominantly non-verbal communication in general practice consultations. $\mathrm{Br} \mathrm{J}$ Gen Pract. 2015;65(635):e351-6

72. Loh A, Simon D, Wills CE, Kriston L, Niebling W, Harter M. The effects of a shared decision-making intervention in primary care of depression: a cluster-randomized controlled trial. Patient Educ Couns. 2007;67(3):324-32.

73. Lonsdale C, Hall AM, Murray A, Williams GC, McDonough SM, Ntoumanis $\mathbf{N}$, et al. Communication skills training for practitioners to increase patient adherence to home-based rehabilitation for chronic low back pain: results of a cluster randomized controlled trial. Arch Phys Med Rehabil. 2017;98(9):1732-43.e7.

74. Luo Z, Goddeeris J, Gardiner JC, Smith RC. Costs of an intervention for primary care patients with medically unexplained symptoms: a randomized controlled trial. Psychiatr Serv. 2007;58(8):1079-86.

75. Maatouk-Burmann B, Ringel N, Spang J, Weiss C, Moltner A Riemann U, et al. Improving patient-centered communication: results of a randomized controlled trial. Patient Educ Couns. 2016;99(1):117-24.

76. Manze MG, Orner MB, Glickman M, Pbert L, Berlowitz D, Kressin NR. Brief provider communication skills training fails to impact patient hypertension outcomes. Patient Educ Couns. 2015;98(2): 191-8.

77. Mercer SW, Fitzpatrick B, Guthrie B, Fenwick E, Grieve E, Lawson $\mathbf{K}$, et al. The CARE Plus study - a whole-system intervention to improve quality of life of primary care patients with multimorbidity in areas of high socioeconomic deprivation: exploratory cluster randomised controlled trial and cost-utility analysis. BMC Med. 2016;14(1):88.

78. Merckaert I, Delevallez F, Gibon AS, Lienard A, Libert Y, Delvaux N, et al. Transfer of communication skills to the workplace: impact of a 38 hour communication skills training program designed for radiotherapy teams. J Clin Oncol 2015;33(8):901-9.

79. Middleton JF, McKinley RK, Gillies CL. Effect of patient completed agenda forms and doctors' education about the agenda on the outcome of consultations: randomised controlled trial. BMJ. 2006;332(7552):123842.

80. Moral RR, Torres LA, Ortega LP, Larumbe MC, Villalobos AR, Garcia JA, et al. Effectiveness of motivational interviewing to improve therapeutic adherence in patients over 65 years old with chronic diseases: a cluster randomized clinical trial in primary care. Patient Educ Couns. 2015;98(8):977-83.

81. Alamo MM, Moral RR, Perula de Torres LA. Evaluation of a patientcentred approach in generalized musculoskeletal chronic pain/ fibromyalgia patients in primary care. Patient Educ Couns. 2002;48(1):23-31.

82. Penner LA, Gaertner S, Dovidio JF, Hagiwara N, Porcerelli J, Markova T, et al. A social psychological approach to improving the outcomes of racially discordant medical interactions. J Gen Intern Med. 2013;28(9): 1143-9.

83. Pill R, Stott NC, Rollnick SR, Rees M. A randomized controlled trial of an intervention designed to improve the care given in general practice to type II diabetic patients: patient outcomes and professional ability to change behaviour. Fam Pract. 1998;15(3):229-35.

84. Rakel D, Barrett B, Zhang Z, Hoeft T, Chewning B, Marchand L, et al. Perception of empathy in the therapeutic encounter: effects on the common cold. Patient Educ Couns. 2011;85(3):390-7.

85. Rask MT, Jensen ML, Andersen J, Zachariae R. Effects of an intervention aimed at improving nurse-patient communication in an oncology outpatient clinic. Cancer Nurs. 2009;32(1):E1-11.

86. Roter D, Rosenbaum J, de Negri B, Renaud D, DiPrete-Brown L, Hernandez $\mathbf{O}$. The effects of a continuing medical education programme in interpersonal communication skills on doctor practice and patient satisfaction in Trinidad and Tobago. Med Educ. 1998;32(2):181-9.

87. Smith RC, Lyles JS, Mettler J, Stoffelmayr BE, Van Egeren LF, Marshall AA, et al. The effectiveness of intensive training for residents in interviewing. A randomized, controlled study. Ann Intern Med. 1998;128(2):118-26.

88. Song MK, Ward SE, Fine JP, Hanson LC, Lin FC, Hladik GA, et al. Advance care planning and end-of-life decision making in dialysis: a randomized controlled trial targeting patients and their surrogates. Am J Kidney Dis. 2015;66(5):813-22.

89. Stewart M, Brown JB, Hammerton J, Donner A, Gavin A, Holliday RL, et al. Improving communication between doctors and breast cancer patients. Ann Fam Med. 2007;5(5):387-94

90. Swanson AJ, Pantalon MV, Cohen KR. Motivational interviewing and treatment adherence among psychiatric and dually diagnosed patients. J Nerv Ment Dis. 1999;187(10):630-5.

91. Thom DH, Bloch DA, Segal ES. An intervention to increase patients trust in their physicians. Stanford Trust Study Physician Group. Acad Med. 1999;74(2): 195-8.

92. Wolf DM, Lehman L, Guinlin R, Zullo T, Hoffman L. Effect of patientcentered care on patient satisfaction and quality of care. J Nurs Care Qual. 2008;23(4):316-21.

93. Wolf D, Lehman L, Quinlin R, Rosenzweig M, Friede S, Zullo T, et al. Can nurses impact patient outcomes using a patient-centered care model? J Nurs Adm. 2008;38(12):532-40.

94. Ackley BJ SB, Ladwig G, Tucker S. Evidence-Based Nursing Care Guidelines: Medical-Surgical Interventions. 1 ed. St. Louis: Mosby Elsevier; 2007.

95. Mohr JJ, Barach P, Cravero JP, Blike GT, Godfrey MM, Batalden PB, et al. Microsystems in health care: part 6. Designing patient safety into the microsystem. Jt Comm J Qual Saf. 2003;29(8):401-8.

96. Shelton RC, Cooper BR, Stirman Sw. The sustainability of evidencebased interventions and practices in public health and health care. Annu Rev Public Health. 2018;39:55-76.

Publisher's Note Springer Nature remains neutral with regard to jurisdictional claims in published maps and institutional affiliations. 